\title{
Evidence-based pelvic floor disorder care pathways optimize shared decision making between patients and surgeons
}

\author{
Lauren Caldwell ${ }^{1}$ (D) Amy E. Papermaster ${ }^{1} \cdot$ Gabriela E. Halder $^{2} \cdot$ Amanda B. White $^{1} \cdot$ Amy Young $^{1}$. \\ Rebecca G. Rogers ${ }^{3}$
}

Received: 23 August 2021 / Accepted: 18 October 2021 / Published online: 10 January 2022

(c) The International Urogynecological Association 2021

\begin{abstract}
Introduction and hypothesis Evidence-based care pathways improve care standardization and patient outcomes. We created pelvic organ prolapse (POP) and stress urinary incontinence (SUI) care pathways as decision aids for our multidisciplinary team to use when counseling patients.

Methods Using a modified Delphi process, an expert team reviewed existing guidelines and literature to reach consensus on pathway definitions and components.

Results Entry to the care pathways occurs via an advanced practice provider visit. Symptom and quality-of-life questionnaires as well as open-ended patient goals are used to guide patient-provider shared decision making. All treatment choices, including surgical and nonsurgical management, are presented to patients by advanced practice providers. Patients electing nonsurgical management follow-up by telehealth (preferred) or in-person visits as determined by the care pathway. Surgeon consultations are scheduled for patients desiring surgery. Surgical patients undergo urodynamics, simple cystometrics or deferred bladder testing according to the urodynamics clinical pathway. Postoperative follow-up includes telehealth visits and minimizes in-person visits for women with uncomplicated postoperative courses. Patients with resolution of symptoms are graduated from clinic and return to their referring physician. The pathways are revised following publication of new compelling evidence.

Conclusions We developed POP and SUI care pathways to standardize care across a diverse provider group. Advanced practice providers use care pathways with patients as shared decision-making tools for initial evaluation of patients with prolapse and incontinence. These pathways serve as components of value-based care and encourage team members to function independently while utilizing the full scope of their training.
\end{abstract}

Keywords Advanced practice provider · Care pathway · Patient-reported outcomes · Pelvic organ prolapse $\cdot$ Pessary · Shared decision making $\cdot$ Stress urinary incontinence $\cdot$ Value-based care

\section{Introduction}

Presented at the Society of Gynecologic Surgeons 47th Annual Scientific Meeting, 27-30 June 2021, Palm Springs, CA, USA

Lauren Caldwell

Lauren.Caldwell@ascension.org

1 University of Texas Dell Medical School, 1301 W. 38th Street, Suite 705, Austin, TX 78705, USA

2 University of Texas Medical Branch, Galveston, TX, USA

3 Albany Medical Center, Albany, NY, USA
Pelvic organ prolapse (POP) and urinary incontinence (UI) are commonly diagnosed conditions. In the United States up to 4.9 million women will be diagnosed with prolapse by 2050, and an estimated 28.4 million are expected to suffer from urinary incontinence [1]. Although $11 \%$ of women will undergo surgery for POP or UI before age 80 , many patients elect to manage these quality-of-life conditions conservatively in the outpatient setting [2]. The urogynecology provider must deliver high-quality and effective care for patients with POP and/or UI using both surgical and nonsurgical interventions. 
Value-based healthcare, defined as improvement in patient-centered outcomes at lower healthcare costs, is an increasingly important consideration in healthcare delivery [3]. Standardized care pathways are used to ensure evidencebased and high-value care and have been shown to improve clinical outcomes [4-7]. Although clinical pathways may incorporate a variety of components, it is generally accepted that a pathway must detail the steps for delivery of structured, multidisciplinary care based on sound evidence for a distinct patient population [8].

To address the urgent need for value-based clinical care, the Women's Health Institute at the Dell Medical School at the University of Texas at Austin adopted a novel integrated practice unit (IPU) model for the care of pelvic floor disorders. The development of a bundled payment model in this IPU clinic has been previously described [9]. The IPU setting encompasses co-located multidisciplinary teams, including both surgeons and advanced practice providers (APPs), as well as ancillary services, such as pelvic floor physical therapy (PFPT), nutrition and social work, improving ancillary service access for a widely varied patient population [10]. The multidisciplinary nature of our IPU, as well as its bundled payment model design, necessitates a uniform method of patient evaluation and treatment amongst providers. We created standardized POP and stress urinary incontinence (SUI) care pathways to be used as decision aids for our care team and patients as a component of value-based care.

\section{Materials and methods}

This work was submitted to the University of Texas IRB as STUDY00000144 and STUDY00000174 and granted a waiver as no human subjects were involved. A multidisciplinary expert team, including generalist obstetricians and gynecologists, urogynecology surgeons, APPs, and pelvic floor physical therapists contributed to the development of the standardized POP care pathway. This team reviewed existing guidelines and literature for POP and SUI diagnosis and treatment, including landmark articles and society opinions, to reach consensus on care pathway definitions and components. A modified Delphi technique was utilized for construction of the care pathways [11]. Using this technique, providers first discussed possible definitions and components of a POP or SUI care pathway in an open-ended fashion. After an initial care pathway was developed, it was presented to the group for revisions and feedback was obtained to edit the pathway. With each variation of the clinical pathway, group feedback was obtained and used to modify the pathway until reliable consensus was reached. The same process was used to design the urodynamics clinical pathway. All pathways underwent external expert review. These pathways were then instituted in the University of Texas at Austin Dell Medical School Women's Health Institute IPU. Pathways are periodically revised by the multidisciplinary team following publication of new compelling evidence or with opportunity for optimizing value-based care.

\section{Results}

\section{Pelvic organ prolapse}

Our POP care pathway is illustrated in Fig. 1. Entry to care occurs with an initial evaluation by an APP. Patient-reported outcomes (PROs) and open-ended patient goals are collected at the time of this visit. PROs refer to several symptom and quality-of-life questionnaires, including the Pelvic Floor Distress Inventory-20 (PFDI-20), Pelvic Organ Prolapse/ Incontinence Sexual Questionnaire-IUGA Revised (PISQIR), General Anxiety Disorder Assessment-7 (GAD-7), and Patient Health Questionnaire-9 (PHQ-9) [12-15]. These questionnaires were selected as the most commonly utilized symptom bother questionnaires in urogynecology studies and mental health screening in the primary care literature. Responses are used to guide patient-provider shared decision making throughout the patient's time under IPU care. A thorough patient history is obtained. Evaluation includes measurement of post-void residual (PVR) and Pelvic Organ Prolapse Quantification (POP-Q) examination. Treatment choices of pessary, PFPT, and surgery are presented to all patients by APPs and one initial option is selected through shared decision making.

The patient and APP may determine that nonsurgical management is appropriate to meet the patient's goals. The option of observation alone is discussed only for patients with stage 0-2 POP as determined by the POP-Q examination. Patients with stage 3 or 4 POP who desire observation are advised to first undergo renal ultrasound and measurement of blood urea nitrogen and creatinine as advanced prolapse can rarely lead to ureteral kinking [16, 17]. Patients electing for nonsurgical management with pessary return for a pessary fitting with an APP, as well as a 2-week follow-up APP visit. Subsequent follow-up varies between self-managed and indwelling pessaries as detailed in Fig. 1 and may be performed by the referring physician or in the IPU if outside of the referring physician's scope of practice. Patients electing for PFPT are scheduled for an initial evaluation and follow-up by a physical therapist $[18,19]$. Subsequent PFPT visits are preferably conducted via telehealth, although they may also be in-person, depending on both the patient and the condition being treated.

If after initial consultation the patient desires surgical management to meet their goals, consultation with a urogynecology surgeon is scheduled. All urogynecology 


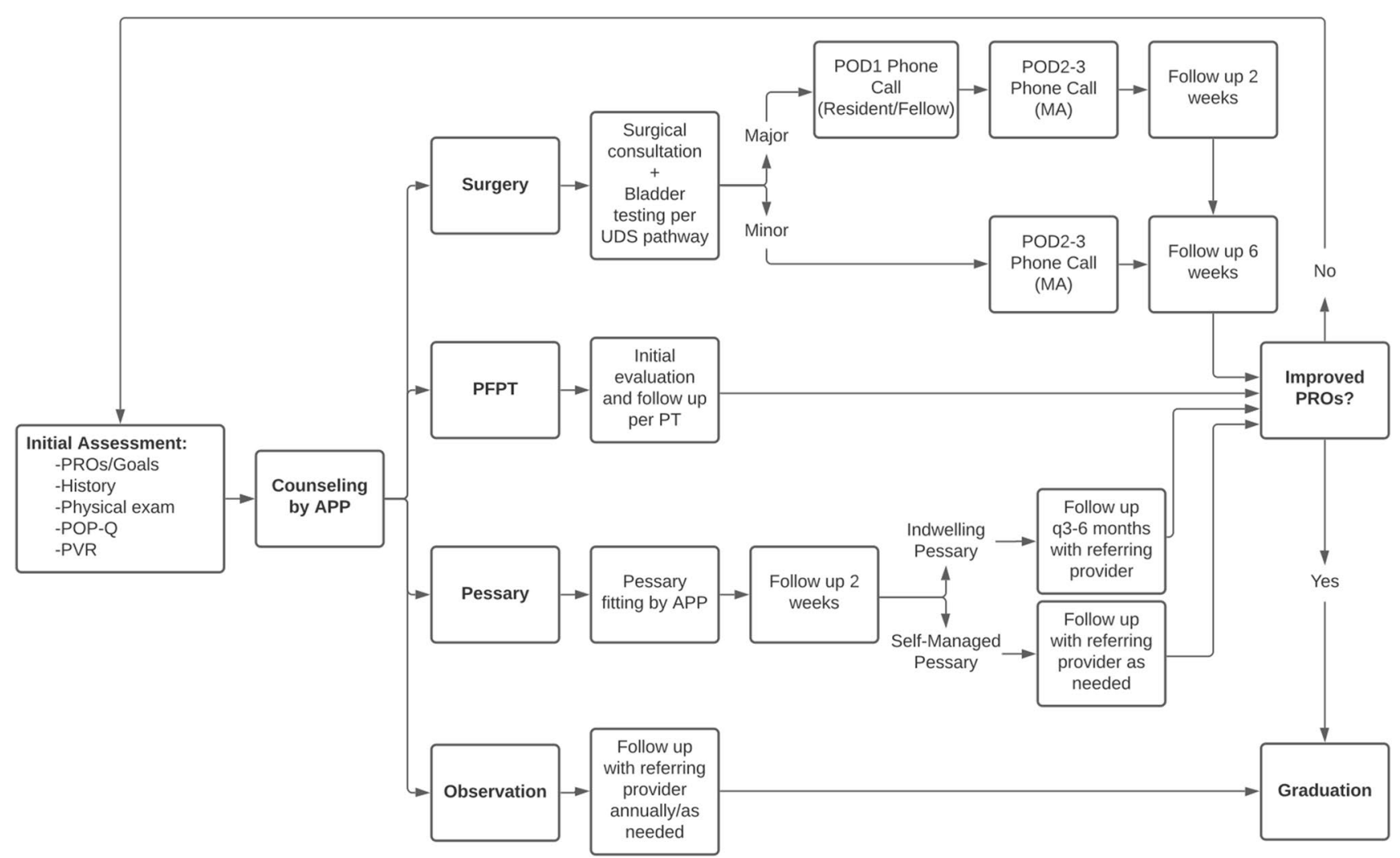

Fig. 1 Pelvic organ prolapse clinical pathway. $A P P$ advanced practice provider, $M A$ medical assistant, $P F P T$ pelvic floor physical therapy, $P O D$ postoperative day, $P O P-Q$ Pelvic Organ Prolapse Quantification, $P R O s$ patient-reported outcomes, $P V R$ postvoid residual, $U D S$ urodynamics

surgeons in our practice offer all routes of prolapse repair, allowing surgical patients to be referred to the first available surgeon. Based on our multidisciplinary expert team's interpretation of existing literature, surgical patients undergo urodynamics (UDS), simple cystometrics (SC), or deferred bladder testing according to the UDS clinical pathway illustrated in Fig. 2. Patients with POP and without urinary incontinence are scheduled for SC, which includes bladder backfill, cough stress test, and simple uroflow. Those with POP as well
Fig. 2 Urodynamics clinical pathway. MUI mixed urinary incontinence, $S U I$ stress urinary incontinence, UI urinary incontinence, UUI urgency urinary incontinence

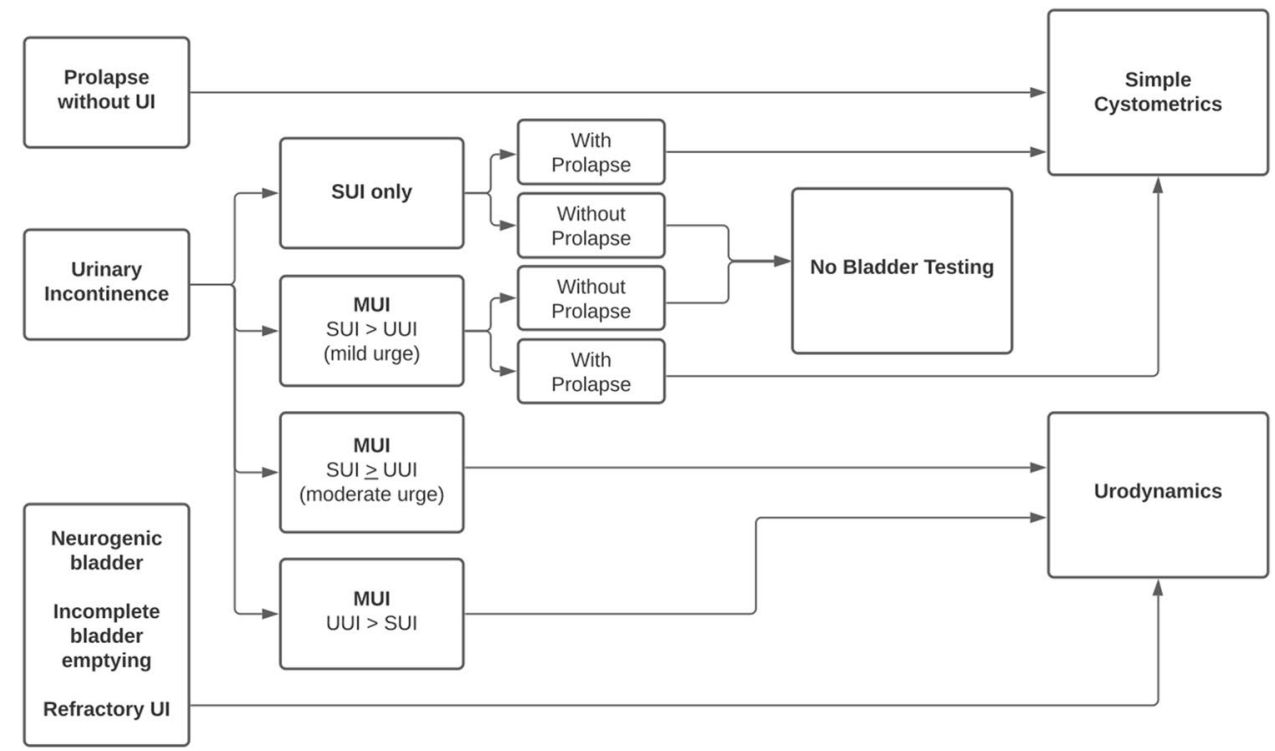


as mixed urinary incontinence (MUI) with mild urge, or SUI also undergo SC. Patients with POP and MUI with predominant or moderate urge are scheduled for UDS [20]. After review of preoperative bladder testing and surgical consultation, patients are scheduled for surgery. Postoperative follow-up is encouraged via telehealth to minimize in-person visits for those with uncomplicated postoperative courses, an update made to our care pathways with emerging evidence during the COVID-19 pandemic [21]. Most surgical patients are seen for at least one in-person postoperative visit. After major surgery, patients are contacted by phone 1 day and 2 weeks after surgery. After minor surgery, patients are contacted by phone 6 weeks after surgery. All postoperative patients are contacted by phone on postoperative day 2 or 3 by a medical assistant (MA).

Following management with pessary, PFPT, or surgery, patients are evaluated for improvement in PROs by APPs. Those with resolution of prolapse symptoms are graduated from the IPU clinic and return to their referring physician. Those with persistently bothersome symptoms undergo repeat counseling and shared decision making is employed to determine the next treatment steps.

\section{Stress urinary incontinence}

Similar to the POP pathway, SUI pathway entry occurs via an APP visit (Fig. 3). PROs are collected at the time of this visit and include the validated symptom and quality-of-life questionnaires previously discussed, as well as open-ended patient goals. These PROs are used to guide patient-provider shared decision making throughout the patient's time under IPU care. A thorough patient history is obtained. Initial evaluation includes urine dip, culture and microscopic urinalysis, and measurement of PVR. Treatment choices of pelvic floor exercises, PFPT, anti-incontinence pessary or surgery are presented and one initial treatment option is selected through shared decision making [19]. Based on a literature review, concurrent use of PFPT and anti-incontinence pessary is not recommended [22].

Patients who elect for nonsurgical management follow-up by telehealth (preferred) or in-person visits as illustrated in Fig. 3. Telehealth visits are preferred based on new evidence published during the COVID-19 pandemic [21]. Incontinence pessaries are managed in the same fashion as pessaries for POP discussed above. Surgeon consultations are scheduled for patients desiring surgical management. Patients with demonstrable SUI do not require UDS [23]. Patients desiring

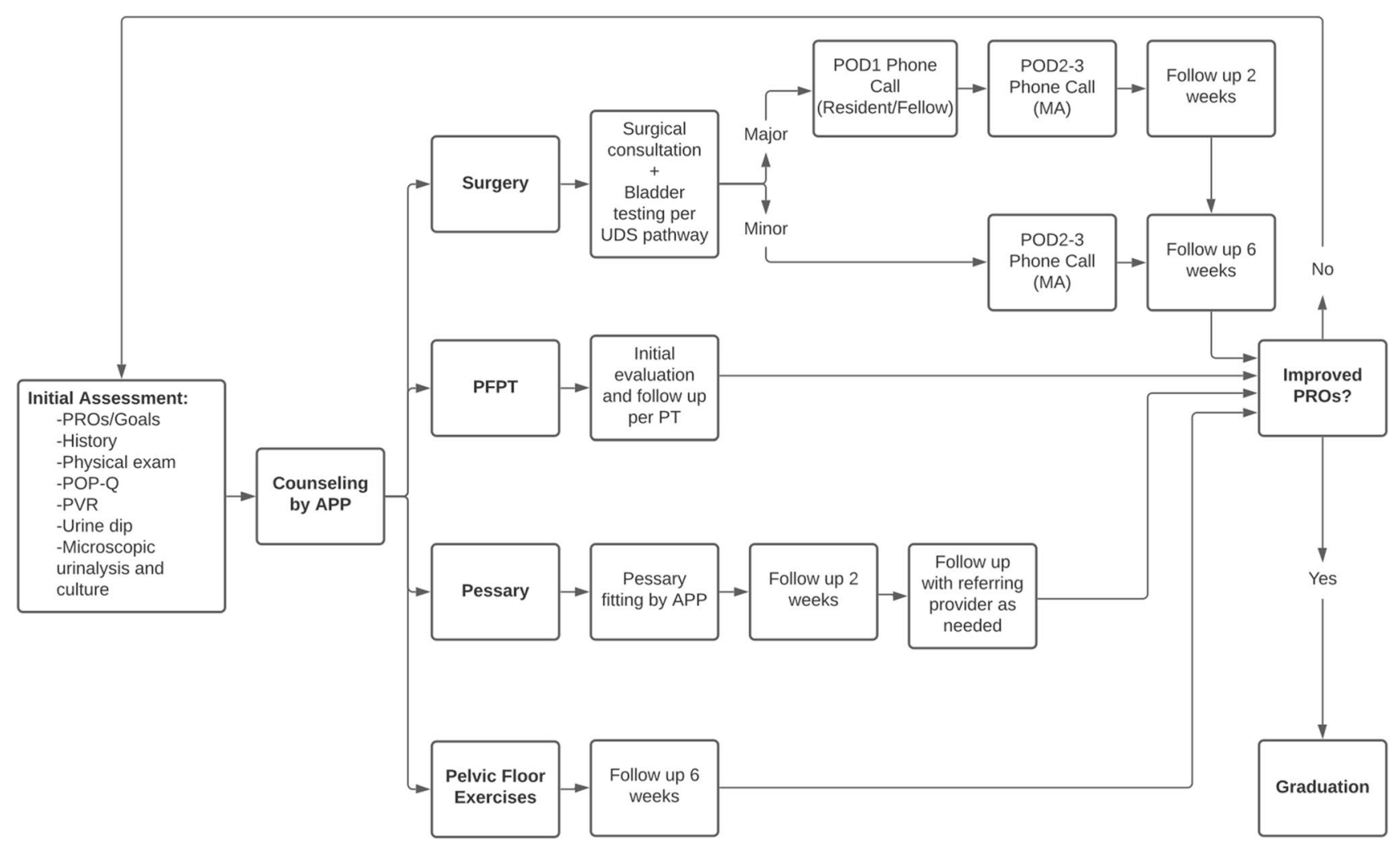

Fig. 3 Stress urinary incontinence clinical pathway. $A P P$ advanced practice provider, $M A$ medical assistant, $P F P T$ pelvic floor physical therapy, $P O D$ postoperative day, $P O P-Q$ Pelvic Organ Prolapse
Quantification, $P R O s$ patient-reported outcomes, $P V R$ postvoid residual, UDS urodynamics 
surgery for SUI without demonstrable SUI on examination undergo either UDS or SC (Fig. 2). Patients with SUI and $\mathrm{POP}$, and those with stress-predominant MUI and no POP undergo SC [24, 25]. After review of preoperative bladder testing and surgical consultation, patients are scheduled for surgery. Postoperative follow-up includes in-person visits and telehealth visits for women with uncomplicated postoperative courses [21]. Patients are contacted by telephone in the same fashion as following POP surgery. Patients with resolution of SUI symptoms are graduated from clinic and return to care with their primary provider. Those with persistently bothersome symptoms undergo repeat counseling and shared decision making is again employed to determine the next steps.

\section{Discussion}

Using a modified Delphi process and expert consensus, our team created evidence-based, standardized care pathways for the management of POP and SUI. These pathways are used as shared decision-making tools between patients and providers in a multidisciplinary integrated practice unit for pelvic floor disorders. They serve as important components of value-based care, with all team members functioning independently and utilizing the full scope of their training.

Strengths of this work include the description of a novel method for standardizing outpatient POP and SUI care. Although surgical care pathways for the treatment of POP and surgical decision aids for SUI have previously been developed, similar pathways for initial evaluation and nonsurgical management of POP and SUI described here are not commonly utilized [26-28]. Second, a clear clinical pathway allows for all care team members to function independently while utilizing the full scope of their training, and to work effectively as a team. All recommended components of the initial patient evaluation, including review of PROs and goals, POP-Q examination, and counseling on treatment options, may be carried out by an experienced APP without direct supervision per American Urogynecologic Society guidelines [29]. This allows the urogynecology surgeon to evaluate primarily surgical patients and complex cases requiring their care. Finally, the pathway promotes value-based care through evidence-based recommendations. Weaknesses include pathway development and implementation at a single institution with a unique integrated practice unit care setting. The authors recognize that their interpretation of available literature for pathway development may differ from other experts in the field, and have attempted to minimize this bias through external expert review. Nonetheless, the pathways may not be easily utilized effectively or without modifications in other practice settings. In addition, patients often present with more than one concurrent pelvic floor disorder, making strict adherence to a single clinical pathway more difficult. Finally, individual and systemic challenges to pathway implementation have been previously described [30].

Clinical care pathways pose several potential advantages with implementation. First, they have been shown to improve clinical outcomes and value. Hospital and intensive care unit length of stay were decreased for patients receiving care according to clinical pathways after blunt thoracic injury [4]. Pediatric patients undergoing cardiac surgery who were managed with an evidence-based care pathway also had a decreased length of hospital stay and overall cost, without an increase in readmission [5]. In addition, clinical pathways improve quality by ensuring evidence-based, efficient, and safe care $[6,7]$. Our proposed pathway further promotes value-based care through an integrated multidisciplinary team, with the initial evaluation and counseling performed by APPs. A recent Cochrane review noted equivalent or improved health outcomes, quality of care, and patient satisfaction with nurse-led care [31]. Independently practicing primary care nurse practitioners have been found to deliver high-quality care with fewer patient emergency room visits [32]. As APPs are increasingly integrated into the urogynecology clinical setting, standardized pathways may further strengthen the quality of patient care and interprofessional collaboration [29].

Our care pathways rely heavily on PROs and patientdirected goals. Patient expectations and perceptions of their pelvic floor disorders and treatment are intimately tied to their individual goals [33]. The achievement of patientdefined goals correlates with an increased likelihood of continuing treatment, such as continuation of pessary use for management of prolapse symptoms [34]. Despite this, PROs are most often utilized as outcome measures in a research setting and less frequently used to guide clinical care [35]. Our pathways provide evidence-based, patient-centered care by allowing PROs and goals to guide clinical decision making. Improved patient retention in a clinic utilizing care navigation pathways for overactive bladder has been demonstrated, suggesting that patients might be satisfied with pathway-driven care [36]. Future cost analyses comparing multidisciplinary clinic use of evidence-based care pathways with a traditional care model would be valuable contributions to the literature on value-based care. Investigation of provider and patient satisfaction with their care and clinical outcomes within a POP and/or SUI pathway are needed. Data collection of these outcome measures is ongoing at our institution.

Our team created evidence-based, standardized care pathways for the evaluation and management of POP and SUI for 
use in our multidisciplinary integrated practice unit. These pathways contribute to the delivery of value-based care for women with pelvic floor disorders.

Contributions L. Caldwell: data collection, manuscript writing/editing; A.E. Papermaster: protocol/project development, data collection; G.E. Halder: protocol/project development, manuscript writing/editing; A.B. White: protocol/project development, data collection, manuscript writing/editing; A. Young: protocol/project development, data collection; R.G. Rogers: protocol/project development, data collection, manuscript writing/editing.

Funding L. Caldwell: none; A.E. Papermaster: none; G.E. Halder: none; A.B. White receives grant funding from Boston Scientific and Medtronic; A. Young receives stipend and travel reimbursement from ACOG and reimbursement for ABOG travel; R.G. Rogers receives royalties from Uptodate, and travel and stipend from ABOG and IUGA.

\section{References}

1. Wu JM, Hundley AF, Fulton RG, Myers ER. Forecasting the prevalence of pelvic floor disorders in U.S. Women: 2010 to 2050. Obstet Gynecol. 2009;114:1278-83.

2. Olsen AL, Smith VJ, Bergstrom JO, Colling JC, Clark AL. Epidemiology of surgically managed pelvic organ prolapse and urinary incontinence. Obstet Gynecol. 1997;89:501-6.

3. Porter ME. Defining and introducing value in health care. In: Evidence-based medicine and the changing nature of health care: 2007 IOM annual meeting summary. Washington, DC: Institute of Medicine; 2008. p. 161-72.

4. Baker E, Woolley A, Xyrichis A, Norton C, Hopkins P, Lee G. How does the implementation of a patient pathway-based intervention in the acute care of blunt thoracic injury impact on patient outcomes? A systematic review of the literature. Injury. 2020;51:1733-43.

5. Lagergren SM, Jensen M, Beaven B, Goudar S. Clinical pathway for the Fontan patient to standardize care and improve outcomes. Cardiol Young. 2020;30:1247-52.

6. Kurtin P, Stucky E. Standardize to excellence: improving the quality and safety of care with clinical pathways. Pediatr Clin North Am. 2009;56:893-904.

7. Brufsky A, Lokay K. How to develop and deliver pathway-based care. Hematol Oncol Clin North Am. 2013;27:843-50.

8. Lawal A, Rotter T, Kinsman L, Machotta A, Ronellenfitsch U, Scott SD, Goodridge D, Plishka C, Groot G. What is a clinical pathway? Refinement of an operational definition to identify clinical pathway studies for a Cochrane systematic review. BMC Med. 2016;14:35.

9. Halder GE, Cardwell J, Gao H, Gardiner H, Nutt S, White A, Young A, Rogers RG. Creating a bundled care payment model for treatment of pelvic floor disorders: introducing value into urogynecology. Am J Obstet Gynecol. 2020;223(4):538-542.e1.

10. Caldwell L, Halder GE, Nutt S, Rogers RG, Wright ML, Baum A, White $\mathrm{AB}$. The use of ancillary services under a bundled care versus a fee-for-service payment model. Female Pelvic Med Reconstr Surg. 2021;27(8):493-6.

11. Hsu CC, Sandford BA. The Delphi technique: making sense of consensus. Pract Assess Res Eval. 2007;12:1-8.

12. Barber MD, Walters MD, Bump RC. Short forms of two condition-specific quality-of-life questionnaires for women with pelvic floor disorders (PFDI-20 and PFIQ-7). Am J Obstet Gynecol. 2005;193(1):103-13.

13. Rogers RG, Rockwood TH, Constantine ML, et al. A new measure of sexual function in women with pelvic floor disorders (PFD): the Pelvic Organ Prolapse/Incontinence Sexual Questionnaire, IUGA-Revised (PISQ-IR). Int Urogynecol J. 2013;24(7):1091-103.

14. Spitzer RL, Kroenke K, Williams JBW, Löwe B. A brief measure for assessing generalized anxiety disorder: the GAD-7. Arch Intern Med. 2006;166(10):1092-7.

15. Kroenke K, Spitzer RL, Williams JBW. The PHQ-9. J Gen Intern Med. 2001;16(9):606-13.

16. Dancz CE, Walker D, Thomas D, Özel B. Prevalence of hydronephrosis in women with advanced pelvic organ prolapse. Urology. 2015;86:250-4.

17. Siddique M, Ingraham C, Kudish B, Iglesia CB, Polland A. Hydronephrosis associated with pelvic organ prolapse: a systematic review. Female Pelvic Med Reconstr Surg. 2020;26:212-8.

18. Hagen S, Stark D, Glazener C, et al. Individualised pelvic floor muscle training in women with pelvic organ prolapse (POPPY): a multicentre randomized controlled trial. Lancet. 2014;383(9919):796-806.

19. Dumoulin C, Hunter KF, Moore K, et al. Conservative management for female urinary incontinence and pelvic organ prolapse review 2013: Summary of the 5th International Consultation on Incontinence. Neurourol Urodyn. 2016;35(1):15-20.

20. American College of Obstetricians and Gynecologists. Pelvic organ prolapse. ACOG Practice Bulletin No. 214. Obstet Gynecol. 2019;134:e126-42.

21. Grimes CL, Balk EM, Crisp CC, et al. A guide for urogynecologic patient care utilizing telemedicine during the COVID19 pandemic: review of existing evidence. Int Urogynecol J. 2020;31(6):1063-89.

22. Richter HE, Burgio KL, Brubaker L, et al. Continence pessary compared with behavioral therapy or combined therapy for stress incontinence: a randomized controlled trial. Obstet Gynecol. 2010;115:609-17.

23. Nager CW, Brubaker L, Litman HJ. A randomized trial of urodynamic testing before stress-incontinence surgery. N Engl J Med. 2012;366:1987-97.

24. Scotti RJ, Myers DL. A comparison of the cough stress test and single-channel cystometry with multichannel urodynamic evaluation in genuine stress incontinence. Obstet Gynecol. 1993;81:430-3.

25. American College of Obstetricians and Gynecologists. Urinary incontinence in women. ACOG Practice Bulletin No. 155. Obstet Gynecol. 2015;126:e66-81.

26. Maher CF, Baessler K, Barber M, et al. Summary: 2017 International Consultation on Incontinence evidence-based surgical pathway for pelvic organ prolapse. Female Pelvic Med Reconstr Surg. 2020;26:30-6.

27. Carter-Brooks CM, Du AL, Ruppert KM, Romanova AL, Zyczynski HM. Implementation of a urogynecology-specific enhanced recovery after surgery (ERAS) pathway. Am J Obstet Gynecol 2018;219:495.e1-10.

28. Ong HL, Sokolova I, Bekarma H, Curtis C, Macdonald A, Agur W. Development, validation and initial evaluation of patientdecision aid (SUI-PDA@) for women considering stress urinary incontinence surgery. Int Urogynecol J. 2019;30:2013-22.

29. A training guide - core competencies for the advanced practice provider entering urogynecology. American Urogynecologic Society 2018.

30. Jabbour M, Newton AS, Johnson D, Curran JA. Defining barriers and enablers for clinical pathway implementation in complex clinical settings. Implement Sci. 2018;13:139. 
31. Laurant M, van der Biezen M, Wijers N, Watananirun K, Kontopantelis E, van Vught AJ. Nurses as substitutes for doctors in primary care. Cochrane Database Syst Rev. 2018;7:CD001271.

32. Traczynski J, Udalova V. Nurse practitioner independence, health care utilization, and health outcomes. J Health Econ. 2018;58:90-109.

33. Hullfish KL, Bovbjerg VE, Gibson J, Steers WD. Patient-centered goals for pelvic floor dysfunction surgery: what is success, and is it achieved? Am J Obstet Gynecol. 2002;187:88-92.

34. Komesu YM, Rogers RG, Rode MA, et al. Patient-selected goal attainment for pessary wearers: what is the clinical relevance? Am J Obstet Gynecol 2008;198:577.e1-5.
35. Rogers RG. Translating patient-reported outcomes to improve patient care and urogynecologic research. Int Urogynecol J. 2019;28:1765-6.

36. Rohloff M, Peifer G, Thompson JH. Patient navigation for overactive bladder improves access to care. Int Urogynecol J. 2020;31:1007-12.

Publisher's note Springer Nature remains neutral with regard to jurisdictional claims in published maps and institutional affiliations. 